\title{
Characterization of Semiconductor Nanospikes Produced by Focused Ion Beam Erosion
}

\author{
K.A. Grossklaus* and J.M. Millunchick* \\ * Materials Science and Engineering Department, University of Michigan, Ann Arbor, MI 48109
}

We report on a focused ion beam (FIB) method for creating III-V semiconductor spike nanostructures. By using a FIB to erode InAs films grown on InP substrates we have been able to create high aspect ratio heterostructure spikes, or "nanospikes," with average heights of $300 \mathrm{~nm}$ and maximum heights greater than $800 \mathrm{~nm}$. The nanospike formation mechanism has been examined in situ by scanning electron microscopy (SEM) observation, and nanospike formation has been found to proceed via an ion-produced droplet masking process. Metallic droplets form on the surface due to preferential sputtering of the group $\mathrm{V}$ material $[1,2]$. These droplets initially move randomly across the surface but as film erosion proceeds they become stationary and act to shield the underlying material. In this way the droplets act as etch-masks that allow nanospikes to form as the surrounding material recedes due to FIB erosion. The droplet etch-masks are replenished by the addition of excess group III atoms produced by sputtering of the surrounding InAs film, and so are self-sustaining [3]. We have found that the InAs/InP film heterostructure is necessary for nanospike formation, as nanospikes will not form on bare InAs or InP substrates upon FIB irradiation. We have also found that the placement of nanospikes is dependent on the initial InAs film morphology and the recession of the InAs/InP interface as erosion proceeds. Nanospikes are constrained by that interface and form on regions of InAs only due to the different FIB responses and excess group III adatom production rates of InAs and InP [2]. Pre-patterning the InAs/InP heterostructure leads to well-ordered arrays of nanospikes. Using a hybrid pre-patterning and FIB erosion approach, it is possible to create ordered arrays of nanospikes in arbitrary patterns. Nanospike structure has been characterized by high resolution transmission electron microscopy (TEM), by scanning transmission electron microscopy (STEM), and by STEM energy dispersive spectrometry (EDS) compositional analysis. Characterization of the nanospike structure by TEM has revealed that they have an ion damaged outer layer and contain a single crystalline core that maintains the structure of the original film and is free of extended defects such as twin boundaries. The electrical I/V response of the nanospikes has been examined using a SEM/nanomanipulator system [4]. Annealing studies have also been conducted to examine the effect that annealing nanospikes under an arsenic overpressure has on their structure and electrical properties.

References

[1] A. Lugstein, M. Weil, B. Basnar, C. Tomastik, and E. Bertagnolli, Nucl. Instr. and Meth. in Phys. Res. B. 222, 91 (2004).

[2] K.A. Grossklaus and J.M. Millunchick, J. Appl. Phys. 109, 014319 (2011).

[3] S. Le Roy, E. Barthel, N. Brun, A. Lelarge, and E. Sondergard, J. Appl. Phys. 106, 094308 (2009).

[4] Electrical characterization was carried out in collaboration with Sandia National Laboratory as part of CINT user proposal U2010B1041. 

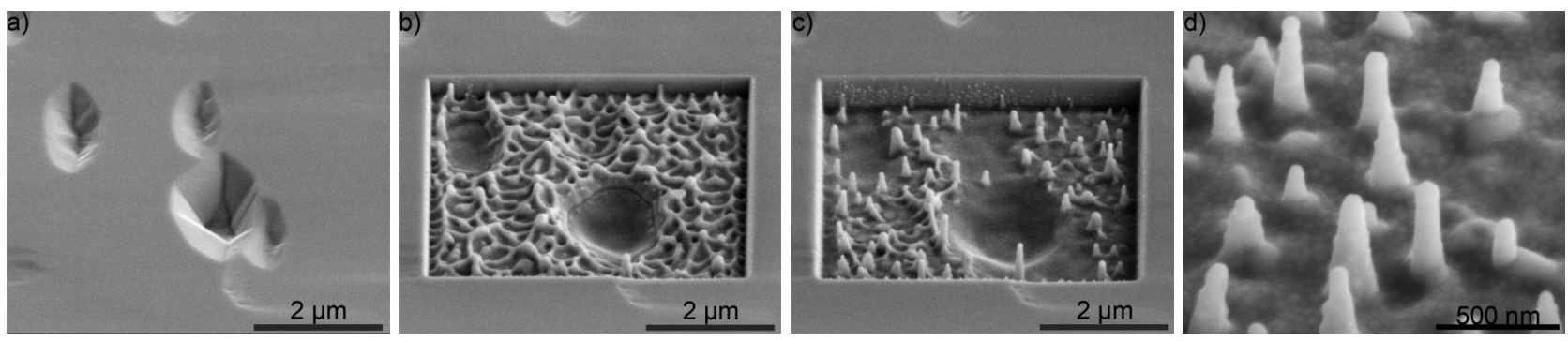

FIG. 1. SEM images showing a region of partially rough InAs film grown on InP through the process of FIB erosion to produce nanospikes. a) shows the region before exposure. b) shows the same location following 7000 ion beam passes, and c) shows the area following 12000 passes. d) shows a higher magnification view of some representative nanospikes. Images were taken with the sample tilted $52^{\circ}$ off the electron beam normal.
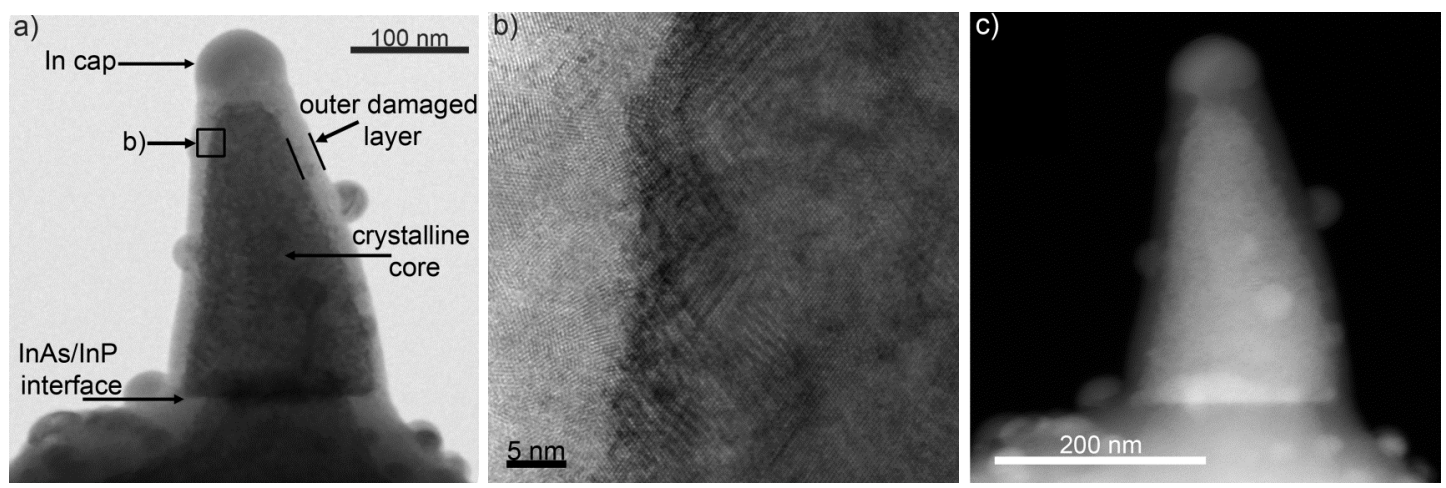

FIG. 2. TEM images of a nanospike. a) shows a $<110>$ bright-field TEM image of a nanospike with the different regions of the nanospike structure labeled. b) is a high resolution TEM image taken at the area indicated in a) showing the boundary between the outer ion damaged layer and the crystalline core of the spike. c) is a HAADF STEM image of the same nanospike which more clearly shows the boundaries between nanospike features and the InAs/InP interface.

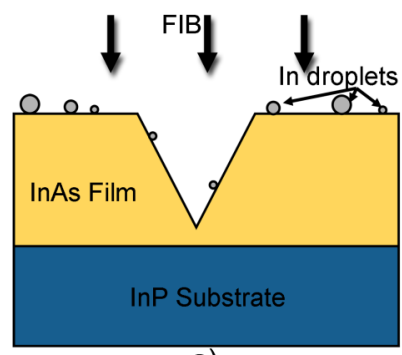

a)

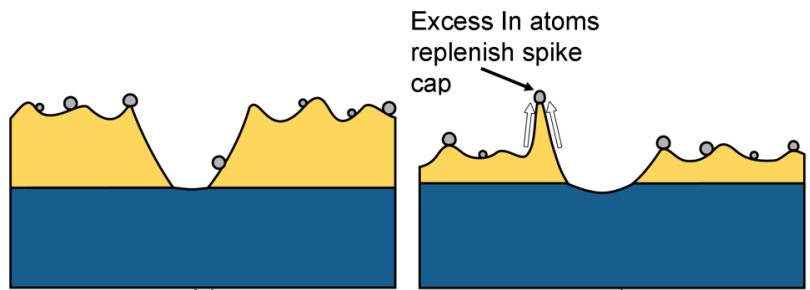

b)

C)

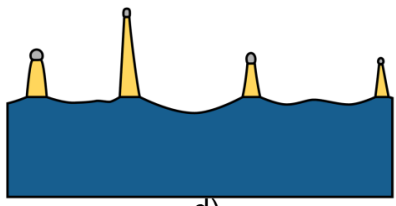

d)

FIG. 3. Schematic detailing the mechanism of nanospike formation. a) FIB irradiation causes Indium droplets to form on a rough InAs film. b) As irradiation continues the InAs surface becomes uneven and rippled. c) An In droplet becomes stationary at an apex of the film and will act as a protective mask for the underlying InAs, allowing a nanospike to form. The mask is replenished by excess In produced as the surrounding InAs erodes. d) More nanospikes will form as other In droplets become stationary until the InAs film is eroded away and the underlying InP substrate is fully exposed. 